\title{
High efficiency light coupling from antiresonant reflecting optical waveguide to integrated photodetector using an antireflecting layer
}

\author{
Toshihiko Baba and Yasuo Kokubun
}

\begin{abstract}
The dispersion characteristics and radiation loss characteristics of ARROW (antiresonant reflecting optical waveguide), a novel single-mode slab waveguide, are analyzed in detail. The mechanism by which the waveguide produces a large amount of light radiation from the waveguide core to the semiconductor substrate is explained and some approximate formulas of propagation characteristics are obtained, which are useful for the design of ARROW. Two novel integrated structures of ARROW and photodetector are proposed to realize high coupling efficiency from waveguide to photodetector, small device size, and easy fabrication process. In these structures, an antireflecting (AR) layer is introduced to greatly enhance coupling efficiency. Their performance is discussed compared with the conventional waveguide structure. It is shown theoretically and experimentally that a high coupling efficiency $(>90 \%)$ is achievable with short detector length in the ARROW structures.
\end{abstract}

\section{Introduction}

The integration of waveguide optical devices on a semiconductor substrate offers the possibility of integrating functional and active optical devices such as laser diodes, photodetectors, branches, wavelength multi/demultiplexers, optical switches, and other electronic circuits. The integration of optical waveguides and photodetectors is of fundamental interest and there have been several studies. ${ }^{1-8}$ When a conventional dielectric waveguide is utilized to couple guided light to a photodetector in the configuration as shown in Fig. $1,1-3$ there exist several problems: (1) it requires a thick cladding layer to reduce the radiation loss resulting from the penetration of the optical field into the high index semiconductor substrate; (2) in the structure shown in Fig. 1(a), both tapered cladding and photodetecting regions must be long enough to achieve low scattering loss and high coupling efficiency, especially in the case of thick core (e.g., same as the core size of single mode fibers: $8-10 \mu \mathrm{m}$ at $\lambda>1.1 \mu \mathrm{m}$ ); (3) in the structure shown in Fig. 1(b), it is difficult to obtain coupling efficiency larger than 50\%; (4) diffraction grating couplers intrinsically have a strong wavelength

The authors are with Yokohama National University, Division of Electrical \& Computer Engineering, 156 Tokiwadai, Hodogayaku, Yokohama 240, Japan.

Received 26 January 1989.

0003-6935/90/182781-12\$02.00/0.

(C) 1990 Optical Society of America. dependence; and (5) sophisticated fabrication techniques are required to form a tapered cladding ${ }^{1,2}$ and/ or grating. ${ }^{3}$ A novel waveguide structure has been needed to overcome these problems.

The antiresonant reflecting optical waveguide, ${ }^{9-11}$ or ARROW (shown in Fig. 2), has been proposed as a novel single mode waveguide utilizing a thin interference cladding sandwiched between the core and the substrate. This cladding consists of two different dielectric films having large index difference such as $\mathrm{Si} /$ $\mathrm{SiO}_{2}$ or $\mathrm{TiO}_{2} / \mathrm{SiO}_{2}$. In previous papers, ${ }^{9,10}$ we showed that the radiation loss of ARROW varies periodically from low loss to maximum value as the thickness of the first cladding layer changes. This is due to the change of the resonant condition of interference cladding layers. Since this waveguide is formed on a semiconductor substrate such as $\mathrm{Si}$, it is advantageous to integrate photodetectors along with other waveguide optical devices.

In the first section of this paper, we describe the propagation characteristics of ARROW in detail. Next, we propose several types of integrated device structures incorporating waveguides and photodetectors using an antireflecting (AR) layer ${ }^{7}$ to enhance the coupling efficiency. The behavior of the radiated light in each structure is analyzed by two different approaches. One is a kind of ray theory, ${ }^{11}$ utilizing a system interference matrix ${ }^{12}$ which is commonly used in the design of multilayer interference filters. This method can provide insight into the physical behavior of each radiation mode. The mechanism by which 


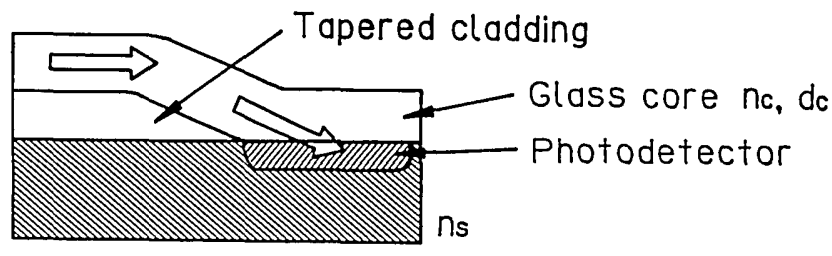

(a)

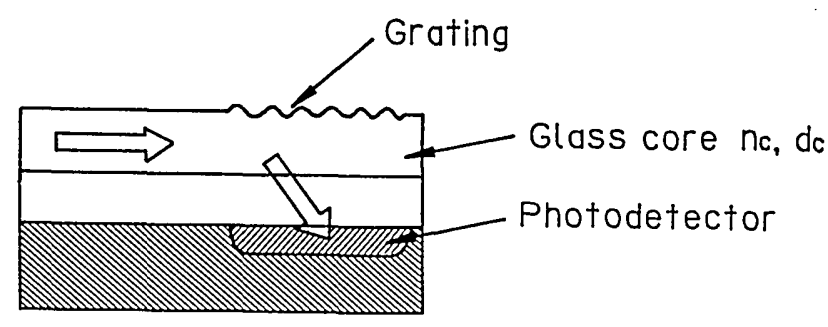

(b)

Fig. 1. Conventional integrated structure of waveguide and photodetector: (a) tapered cladding type; (b) grating type.

ARROW produces a high radiation loss is elucidated by this analysis. The other is an exact wave analysis using a quasi-guided mode theory. ${ }^{13}$ The ARROW integrated structure is compared with the conventional tapered waveguide structure shown in Fig. 1(a). Next, the radiation loss of ARROW is measured experimentally and compared with theoretical values. Discussion is limited to TE polarization because only TE modes can propagate with low loss in ARROW. ${ }^{11}$

\section{Basic Propagation Characteristics of ARROW}

The fundamental structure of ARROW is shown in Fig. 2. An interference cladding, which consists of two films, i.e., a high index layer (the first cladding) and a low index layer (the second cladding) is sandwiched between a core and a semiconductor substrate. The refractive index and thickness of each layer are denoted in the figure.

The material of the core and the second cladding layer is the same $\left(n_{c}=n_{2}\right) ; \mathrm{SiO}_{2}(n=1.46)$ is assumed for these layers in this paper. The material of the first cladding layer must have a refractive index much higher than that of the core and the second cladding layer; $\mathrm{Si}, \mathrm{TiO}_{2}, \mathrm{Al}_{2} \mathrm{O}_{3}, \mathrm{GeO}_{2}, \mathrm{ZrO}_{2}$, etc. can be used for this layer. Other dielectric materials, such as $\mathrm{TiO}_{2}$, are more suitable than $\mathrm{Si}$ in the wavelength region $<0.9$ $\mu \mathrm{m}$, since $\mathrm{Si}$ absorbs in this wavelength region; $\mathrm{TiO}_{2}\left(n_{1}\right.$ $=2.3$ ) is sometimes assumed in this paper.
Light propagates through the $\mathrm{SiO}_{2}$ core by total internal reflection at the upper air- $\mathrm{SiO}_{2}$ boundary and ultra-high reflection at the interference cladding. The reflectivity of the interference cladding is close to unity (>99.9\%), when the thicknesses of interference cladding layers satisfy the antiresonant condition. ${ }^{9-11}$ There exist several leaky modes that are confined to the ARROW core. Only the fundamental mode can propagate with low loss virtually by the loss discrimination of higher order modes, because the mode angles of higher order modes are larger than that of fundamental mode and the reflectivity of interference cladding decreases rapidly with the increase of the propagation angle. ${ }^{9-11}$ Since the refractive index of the first cladding layer is much higher than that of the core and the second cladding, this layer can maintain several modes according to its $V$ parameter. These modes cannot easily be guided because they suffer large scattering loss due to the small thickness of this layer, typically of the order of $0.1 \mu \mathrm{m}$, and also due to the large refractive index difference.

The phase condition of $\nu$ th order mode is generally expressed by ${ }^{10,11}$

$$
2 k_{0} n_{c} d_{c} \sin \theta+\phi_{1}+\phi_{2}=2 \pi \nu,
$$

where $\phi_{1}$ is the phase shift at the core-air boundary and $\phi_{2}$ is the phase shift at the interference cladding, $\theta$ is the propagation angle of light, and $k_{0}$ is the wave number in a vacuum. The propagation constant $\beta_{y}$ and radiation loss coefficient $\alpha_{\nu}$ are given in terms of $\theta$ by

$$
\begin{aligned}
& \beta_{\nu}=k_{0} n_{c} \cos \theta, \\
& \alpha_{\nu}=2.17(1-R) \tan \theta / d_{c e}(\mathrm{~dB} / \mathrm{m}),
\end{aligned}
$$

where $R$ is the power reflectivity of interference cladding and $d_{\mathrm{ce}}$ is the equivalent thickness of the core involving the penetration of light power into the layers outside the core. The power reflectivity is expressed as the squared absolute value of the complex field reflectivity, which depends on the propagation angle $\theta$. Since the phase shifts $\phi_{1}$ and $\phi_{2}$ are obtained as the phase part of the complex field reflectivity of the cladding, the propagation angle $\theta$ can be obtained by solving simultaneously Eq. (1) and the complex field reflectivity with respect to $\theta^{10,11}$; while, $\beta_{\nu}$ and $\alpha_{\nu}$ are calculated numerically from Eqs. (2) and (3), respectively, using $\theta$ so obtained.11

The equivalent index of the guided light $n_{\text {eq }}$ is defined by

$$
n_{\mathrm{eq}} \equiv \beta_{\nu} / k_{0}
$$

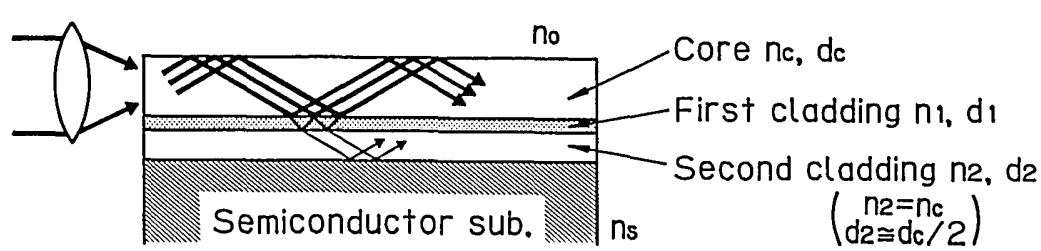

Fig. 2. Fundamental structure of ARROW. 


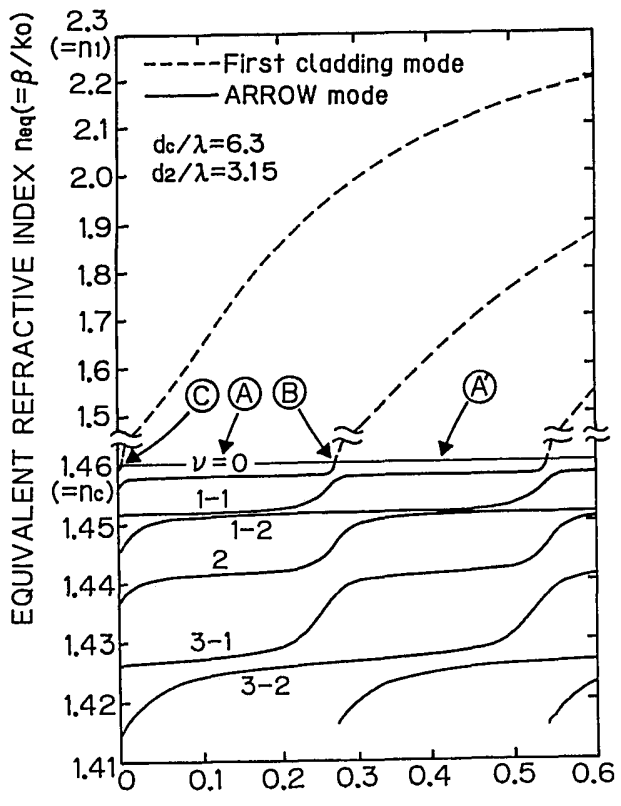

THICKNESS OF FIRST CLADDING $d 1 / \lambda$

(a)

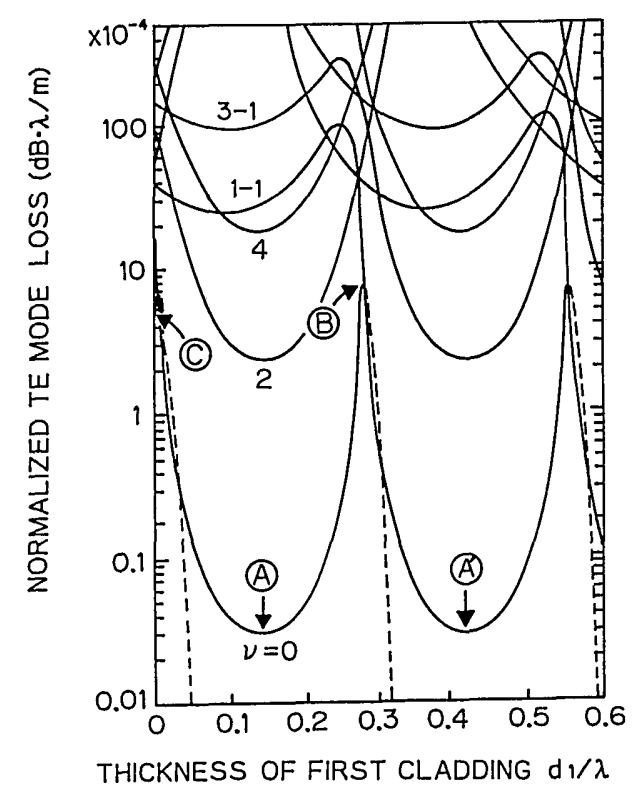

(b)

Fig. 3. Propagation characteristics of ARROW vs thickness of first cladding layer normalized by wavelength: (a) equivalent refractive index characteristic. The values of $d_{c} / \lambda=6.3$ and $d_{2} / \lambda=3.15$ correspond to thicknesses $d_{c}=4 \mu \mathrm{m}$ and $d_{2}=2 \mu \mathrm{m}$ at $\lambda=0.633 \mu \mathrm{m}$, respectively; and (b) propagation loss characteristic.

Figure 3 shows the propagation characteristics of TE modes of ARROW vs the normalized thickness of the first cladding layer $d_{1} / \lambda$. Other thicknesses are also normalized by wavelength. Here, we classify the modes of ARROW into three types as follows:

$$
\left.\begin{array}{rl}
\text { First cladding modes: } & n_{\mathrm{c}}<n_{\mathrm{eq}}<n_{1} \\
\text { ARROW modes: } & n_{0}<n_{\mathrm{eq}}<n_{c} \\
\text { Radiation modes: } & 0<n_{\mathrm{eq}}<n_{0}
\end{array}\right\} \text {, }
$$

where $n_{0}$ is the refractive index of the upper cladding layer and is 1.0 in the case of air, as shown in Fig. 2. In Figs. 3(a) and (b), ARROW modes are drawn by solid lines and first cladding modes are drawn by dashed lines. We give mode numbers independently for the first cladding modes and for the ARROW modes. In the physical meaning, the order of the modes should be numbered according to $n_{\mathrm{eq}}$ in the whole range $0<n_{\mathrm{eq}}<$ $n_{1}$. However, since our main interest here is concentrated on the practical use of ARROW, i.e., the lowest order ARROW mode which propagates with low radiation loss, we numbered the ARROW modes independently of the numbering system of first cladding modes.

Figures 4(a) and (b) display the calculated field distributions of first cladding modes and the lowest order ARROW mode when the thickness of the first cladding layer takes the values indicated by symbols (A) and (A) in Fig. 3, respectively. It is easily seen from these figures that the field of first cladding modes is confined strongly into the thin first cladding layer. On the other hand, most of the field of ARROW mode is confined inside the core of ARROW and the mode size is quite different from that of the first cladding mode. The field of ARROW mode has the number of nodes (zeros) in the vicinity of the first cladding layer corresponding to the order of antiresonant condition defined by Eq. (6). However, the field inside the core is almost the same irrespective of the order of antiresonant condition, i.e., both fields have one maximum inside the core. Thus, it is convenient to unify the ARROW modes having the same number of field maximums inside the core, even under different order antiresonant conditions. In the following, we call the lowest order ARROW mode the fundamental mode.

In Fig. 3(a), the equivalent refractive index of ARROW modes is almost constant over most of the range of $d_{1} / \lambda$. However, it changes abruptly near the cutoffs of the first cladding modes. Beyond these points, the fundamental mode of ARROW changes to a first cladding mode for which the corresponding curve is similar to the typical dispersion characteristics of conventional waveguides. Similarly, each higher order ARROW mode transits to another ARROW mode having an order which is lower by one than the former ARROW mode. These features appear periodically against $d_{1} /$ $\lambda$ so that, in our numbering system, we need to renumber the ARROW modes at the cutoffs of the first cladding modes. Also, we can see that the two curves of odd-order ARROW modes are closely spaced in the range between cutoffs. We numbered these nearly degenerate modes, for example, as 1-1 and 1-2, because both of them have one significant node of light power 


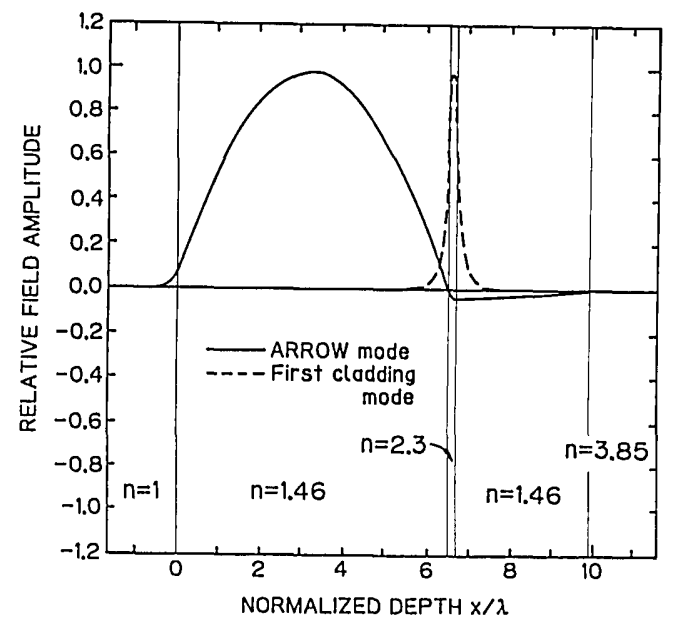

(a)

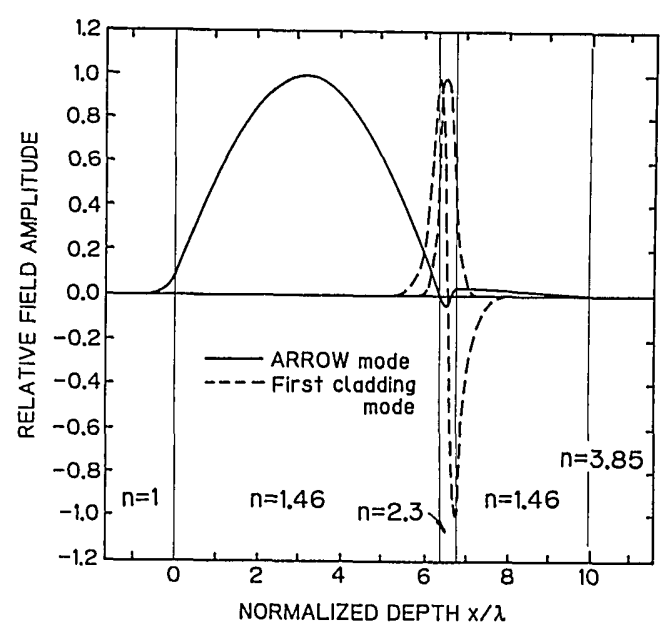

(b)

Fig. 4. Calculated field distribution of first cladding mode and the lowest order ARROW mode: (a) in first-order antiresonant conditions; (b) in second-order antiresonant conditions.

inside the core. The degeneracy of these modes is caused by the light coupling between the core and the second cladding layer.

Figure 3(b) shows the propagation loss characteristics vs the normalized thickness $d_{1} / \lambda$. In this figure, the ordinate is the propagation loss per unit length normalized by wavelength. As $d_{1} / \lambda$ increases, the radiation loss changes periodically. Mode numbers are renumbered in the same way as in Fig. 3(a). The thickness of the first cladding layer at which the loss of ARROW mode takes its minimum value is given by the antiresonant condition ${ }^{9-11}$ :

$$
\frac{d_{1}}{\lambda} \simeq \frac{1}{4 n_{1}}\left[1-\left(\frac{n_{c}}{n_{1}}\right)^{2}+\left(\frac{\lambda}{2 n_{1} d_{\mathrm{ce}}}\right)^{2}\right]^{-1 / 2}(2 N+1),
$$

where $N=0,1,2, \ldots$, and $d_{c e}$ of the fundamental mode of ARROW is approximately given by ${ }^{11}$

$$
d_{\mathrm{ce}} \simeq d_{c}+\frac{\lambda}{2 \pi \sqrt{n_{c}^{2}-n_{0}^{2}}} \text {. }
$$

An extremely low loss of $0.05 \mathrm{~dB} / \mathrm{cm}$ is achievable at $\lambda$ $=0.633 \mu \mathrm{m}$ [indicated by symbols (A) and (A) in Fig. 3(b)], when we assume $\mathrm{TiO}_{2}$ for the first cladding layer. The low loss range is fairly broad. On the other hand, the loss takes its maximum value (indicated by symbol (B)) when $d_{1} / \lambda$ approaches the value given by

$$
\frac{d_{1}}{\lambda} \simeq \frac{1}{2 n_{1}}\left[M+\frac{3 \lambda}{2 \pi^{2} d_{\mathrm{ce}} \sqrt{n_{1}^{2}-n_{c}^{2}}}\right]\left[1-\left(\frac{n_{c}}{n_{1}}\right)^{2}\right]^{-1 / 2},
$$

$$
(M=0,1,2, \ldots) \text {. }
$$

We call the condition expressed by Eq. (8) the resonant condition of order $M$. The thickness given by Eq. (8) is identical to the cutoff condition of $M$ th first cladding mode. This complicated form is derived from the asymmetric structure with respect to the first cladding layer (see Appendix A). As $d_{1} / \lambda$ becomes larger than the value of Eq. (8), most of the light power is confined in the first cladding layer and the radiation loss decreases rapidly along the dashed curve in Fig. 3(b).

At the values of $d_{1}$ given by Eqs. (6) and (8), minimum and maximum loss coefficients of fundamental mode normalized by wavelength, are approximately expressed as follows:

$$
\begin{aligned}
& \alpha_{0 \min } \lambda \simeq 0.543\left(\frac{\lambda}{d_{\mathrm{ce}}}\right)^{5} /\left\{n_{c}\left(n_{1}^{2}-n_{c}^{2}\right) \sqrt{n_{s}^{2}-n_{c}^{2}}\right\}(\mathrm{dB} \cdot \lambda / \mathrm{m}), \\
& \alpha_{0 \max } \lambda \simeq 0.879\left(\frac{\lambda}{d_{\mathrm{ce}}}\right)^{3} /\left(n_{c} \sqrt{n_{s}^{2}-n_{c}^{2}}\right)(\mathrm{dB} \cdot \lambda / \mathrm{m}) .
\end{aligned}
$$

Equation (9) implies that minimum loss strongly depends on the ratio of core thickness to wavelength and also on the relative index differences between the core and the first cladding layer and between the core and the substrate. On the other hand, we derived Eq. (10) using the cutoff condition of the first cladding modes (see Appendix B). Equation (10) includes only dependence on the parameters of the core and the substrate only. In normal ARROW, ${ }^{9-11}$ the maximum $\alpha_{0}$ reaches a very large value, typically several hundred times as large as the minimum value given by Eq. (9).

Similar to Eq. (10), we derived the formula for the loss coefficient at $d_{1}=0$ (indicated by symbol (C) in Fig. 3(b)) as follows:

$$
\alpha_{0}\left(d_{1}=0\right) \lambda \simeq 0.643\left(\frac{\lambda}{d_{\mathrm{ce}}}\right)^{3} /\left(n_{\mathrm{c}} \sqrt{n_{s}^{2}-n_{\mathrm{c}}^{2}}\right)(\mathrm{dB} \cdot \lambda / \mathrm{m}) .
$$

The coefficients in Eq. (10) is a little larger than that in Eq. (11) because the interference of the first cladding layer extinguishes the reflection under the core effectively.

\section{Integrated Structures of Waveguide and Photodetector}

Let us consider three types of integrated structures of waveguide and photodetector as shown in Figs. 5(a)- 


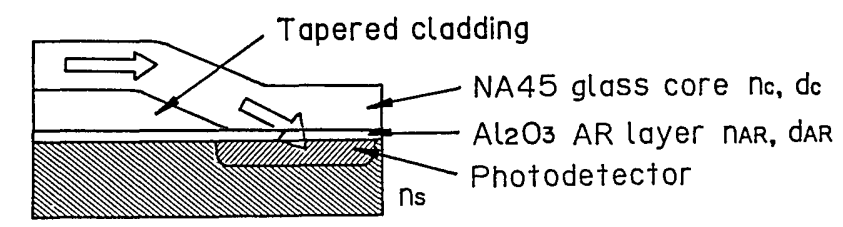

(a)

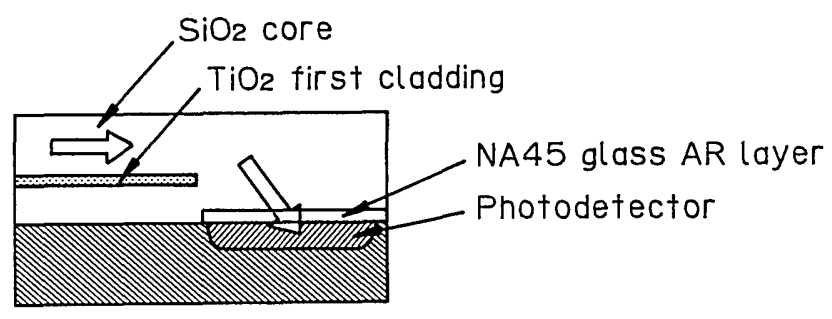

(b)

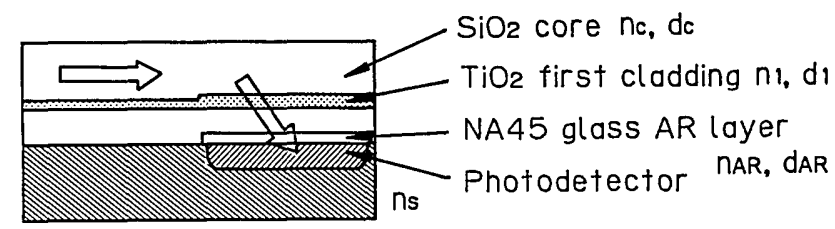

(c)

Fig. 5. Integrated structures of waveguide and photodetector: (a) conventional waveguide structure in which an antireflecting (AR) layer is arranged on the photodetector to enhance coupling efficiency; (b) punch-through ARROW structure with AR layer (the first cladding layer above the photodetector is simply removed); and (c) resonant ARROW structure with AR layer (thickness of first cladding layer is adjusted to resonant conditions).

(c). Figure 5(a) shows a conventional waveguide structure. In this type, the core, having a refractive index a little higher than the cladding, approaches the substrate gradually through the tapered cladding portion. This type of waveguide is regarded as a simple leaky waveguide on the surface of the photodetector. The problem of this type is that the tapered cladding must be long enough to maintain low scattering loss, as mentioned in Sec. I.

Figure 5(b) shows an ARROW structure for which the first cladding layer above the photodetector is removed. This structure is easy to fabricate, and the coupling efficiency to the photodetector has no wavelength dependence. Figure 5(c) shows a similar ARROW structure. The difference from Fig. 5(b) is that the thickness of first cladding layer in the photodetecting region satisfies the condition given by Eq. (8). We can see from Eq. (8) that this type has a strong wavelength dependence corresponding to the resonant condition of the interference cladding. This dependence has been applied to a monolithic integration of demultiplexer and photodetector. ${ }^{7}$ These ARROW structures do not require additional length in the tapered cladding. In the following part of this paper, we will use the symbols [a]-[c] to distinguish these structures according to their designation in the figures.

The coupling efficiency from waveguide to photode-

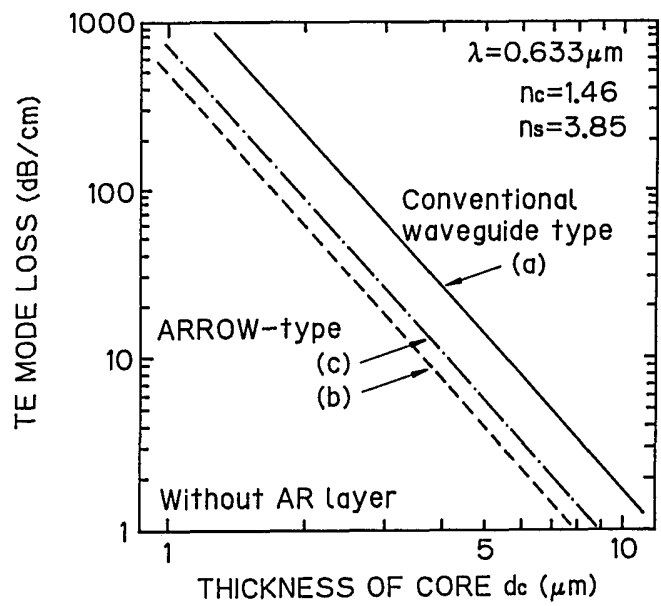

Fig. 6. Calculated maximum radiation loss of integrated structures without AR layer vs thickness of core at $\lambda=0.633 \mu \mathrm{m}$.

tector is identical to the radiation loss from the waveguide to the substrate. For example, a radiation loss coefficient of $100 \mathrm{~dB} / \mathrm{cm}$ is required to obtain a coupling efficiency of $90 \%$ for a detecting length of $1 \mathrm{~mm}$. For ARROW structure [c], the loss coefficient in the photodetecting region is given by Eq. (10). It is given by Eq. (11) for ARROW structure [b]. - We derived a similar approximate formula for conventional waveguide structure [a] as follows:

$$
\alpha_{0} \lambda \simeq 2.170\left(\frac{\lambda}{d_{\mathrm{ce}}}\right)^{3} /\left(n_{\mathrm{c}} \sqrt{n_{s}^{2}-n_{c}^{2}}\right)(\mathrm{dB} \cdot \lambda / \mathrm{m}),
$$

where a simple leaky waveguide is considered, in which the core is sandwiched between the air and the substrate, and $d_{\mathrm{ce}}$ is given by Eq. (7). This coefficient is 2.47 times as large as that of ARROW structures, which is derived from Eq. (10). As an example, loss figures calculated by Eqs. (10)-(12) are plotted vs the thickness of core $d_{c}$ in Fig. 6, assuming $\mathrm{SiO}_{2}$ as the core and $\mathrm{Si}$ as the substrate at $\lambda=0.633 \mu \mathrm{m}$.

Next, we propose to introduce an antireflecting (AR) layer on the surface of the photodetector to enhance the radiation loss. Optimum refractive index $n_{\mathrm{AR}}$ and thickness $d_{\mathrm{AR}}$ of the AR layer are given approximately by

$$
\begin{aligned}
& n_{\mathrm{AR}}^{2}=n_{c}^{2} \cos ^{2} \theta+n_{c} \sin \theta \sqrt{n_{s}^{2}-n_{c}^{2} \cos ^{2} \theta,} \\
& \frac{d_{\mathrm{AR}}}{\lambda} \simeq \frac{1}{4 n_{\mathrm{AR}}}\left[1-\left(\frac{n_{c}}{n_{\mathrm{AR}}}\right)^{2} \cos ^{2} \theta\right]^{-1 / 2} \times(2 I+1), \\
& \quad(I=0,1,2, \ldots),
\end{aligned}
$$

where $\theta$ is the propagation angle of guided light. Angle $\theta$ is usually in the range from $2-4^{\circ}$ when the normalized core thickness $d_{c} / \lambda$ is $5-8$ (e.g., $4 \mu \mathrm{m}$ at $\lambda=0.633 \mu \mathrm{m}, 8$ $\mu \mathrm{m}$ at $\lambda=1.3 \mu \mathrm{m})$. Under this condition, $n_{\mathrm{AR}}$ is calculated as 1.5-1.6 so that glasses such as NA45 glass (made by HOYA Corp., $n=1.54$ ) and $\mathrm{Al}_{2} \mathrm{O}_{3}(n=1.62$ ) are suitable for this layer. When the cladding layer is thick enough in structure [a], we need not etch the AR layer on the substrate outside the surface of photode- 
tecting region. On the other hand, in ARROW structures, the AR layer must be limited to the region on the photodetector, because the reflection at the interface between the second cladding layer and the substrate also plays an important role in the low loss propagation.

\section{Effect of AR Layer}

In the previous sections, we described the calculation of propagation constant and loss by solving the phase equation (1). However, those propagation characteristics do not provide detailed information on the practical behavior of light in the coupling portion where light is radiated to the photodetector, because the previous analysis does not take into account the power distribution of each eigenmode. To express precisely the transitional behavior of light power when the light is incident on the coupling portion from a low loss waveguide, the light should be expressed by the superimposition of many radiation modes into which the input light power is expanded according to the excitation factors. Particularly in the structure having the AR layer, the mode distribution becomes more complicated and ambiguous. In this section, we describe an exact wave analysis of each structure by the quasiguided mode theory and evaluate the effect of the AR layer.

The model of ARROW which we use here is illustrated in Fig. 7. With the aid of the characteristic mode expansion, ${ }^{13}$ one of the field components $E_{y}(x, z)$ is given by

$$
E_{y}(x, z)=\int_{-\infty}^{\infty} L(\beta) N^{2}(\beta) f(x ; \beta) \exp (-j z d \beta) d \beta,
$$

where $f(x ; \beta)$ is the field function of an eigenmode determined by the waveguide structure, $L(\beta)$ is the excitation factor determined by the field distribution of incident light, and $N(\beta)$ is a normalization factor defined in terms of the time-averaged Poynting power. ${ }^{13,14}$ The transition of light power can be calculated by the integration of power at each position $x$. (Detailed calculations are presented in Appendix C.)

Figure 8 shows the power decay inside the core measured in $\mathrm{dB}$ vs the propagation distance normalized by the wavelength. Figure 8(a) shows the characteristic of the conventional waveguide structure [a] with the AR layer for various core thicknesses. Here, the fundamental mode in the leaky waveguide without cladding and AR layers was assumed for the field of input light, $E_{y}(x, 0)$. When the size of core is $<2 \lambda$, the light vanishes instantly within a propagation distance shorter than $100 \lambda$. Even when $d_{c} / \lambda=6-10$, the required length to obtain the coupling efficiency $\eta>90 \%$ is only $400 \lambda$, which is approximately $1 / 67$ of that without the AR layer calculated from Eq. (12). Figure 8 (b) shows the comparison between the characteristics of the three structures. Here, we assume a typical core size of $d_{c} / \lambda=6.3$, which is equivalent to $d_{c}=4 \mu \mathrm{m}$ at $\lambda$ $=0.633 \mu \mathrm{m}$ and $d_{c}=8 \mu \mathrm{m}$ at $\lambda=1.3 \mu \mathrm{m}$. We can see that the ARROW structure [c] can achieve power decay as quickly as the conventional waveguide structure

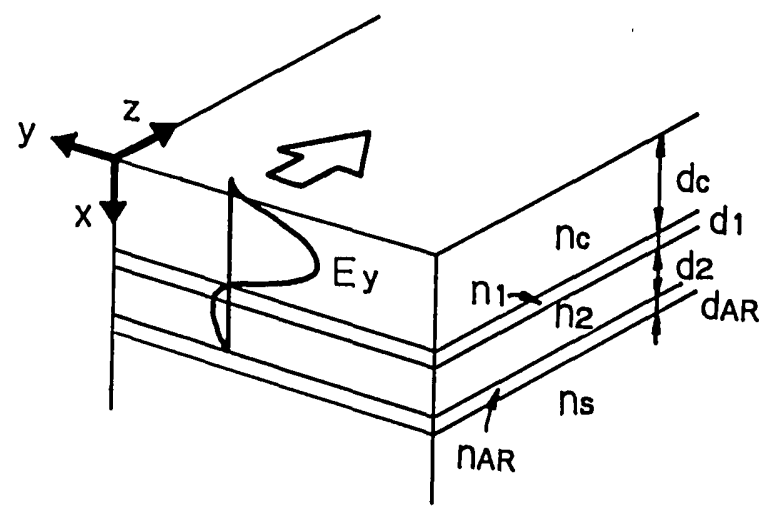

Fig. 7. Wave analysis model of ARROW. Same model can be adapted to conventional waveguide structure by putting $d_{1}=0$ and $d_{2}=0$.

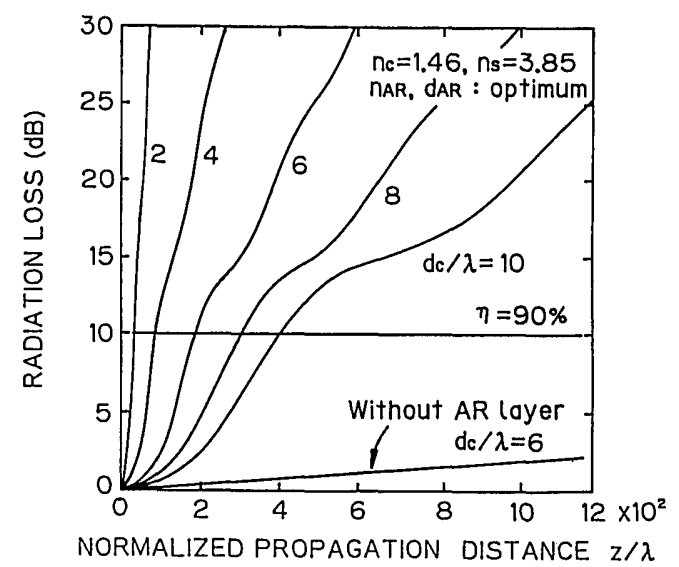

(a)

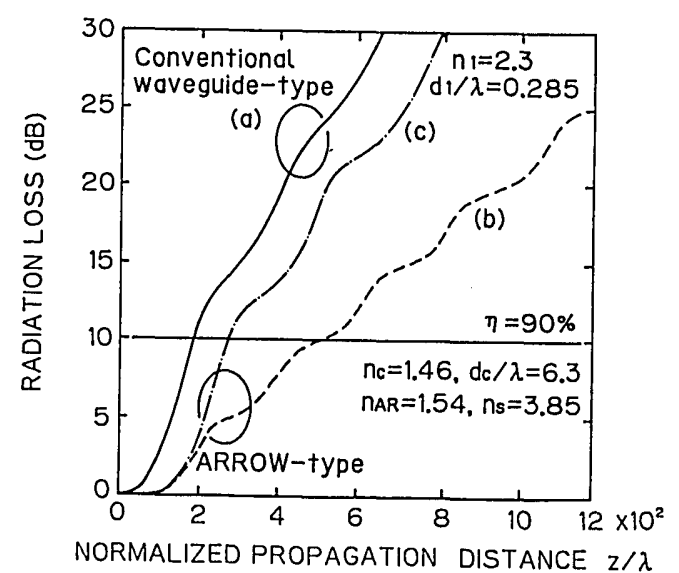

(b)

Fig. 8. Calculated radiation loss vs propagation distance. Ideal AR layer is assumed for each structure. Length of tapered cladding in conventional waveguide structure is not included in the distance. (a) Conventional waveguide structure for various thickness of core $d_{c}$. Results without AR layer for $d_{c} / \lambda=6$ added in contrast with that with AR layer. (b) Comparison among ARROW structures and conventional waveguide. 


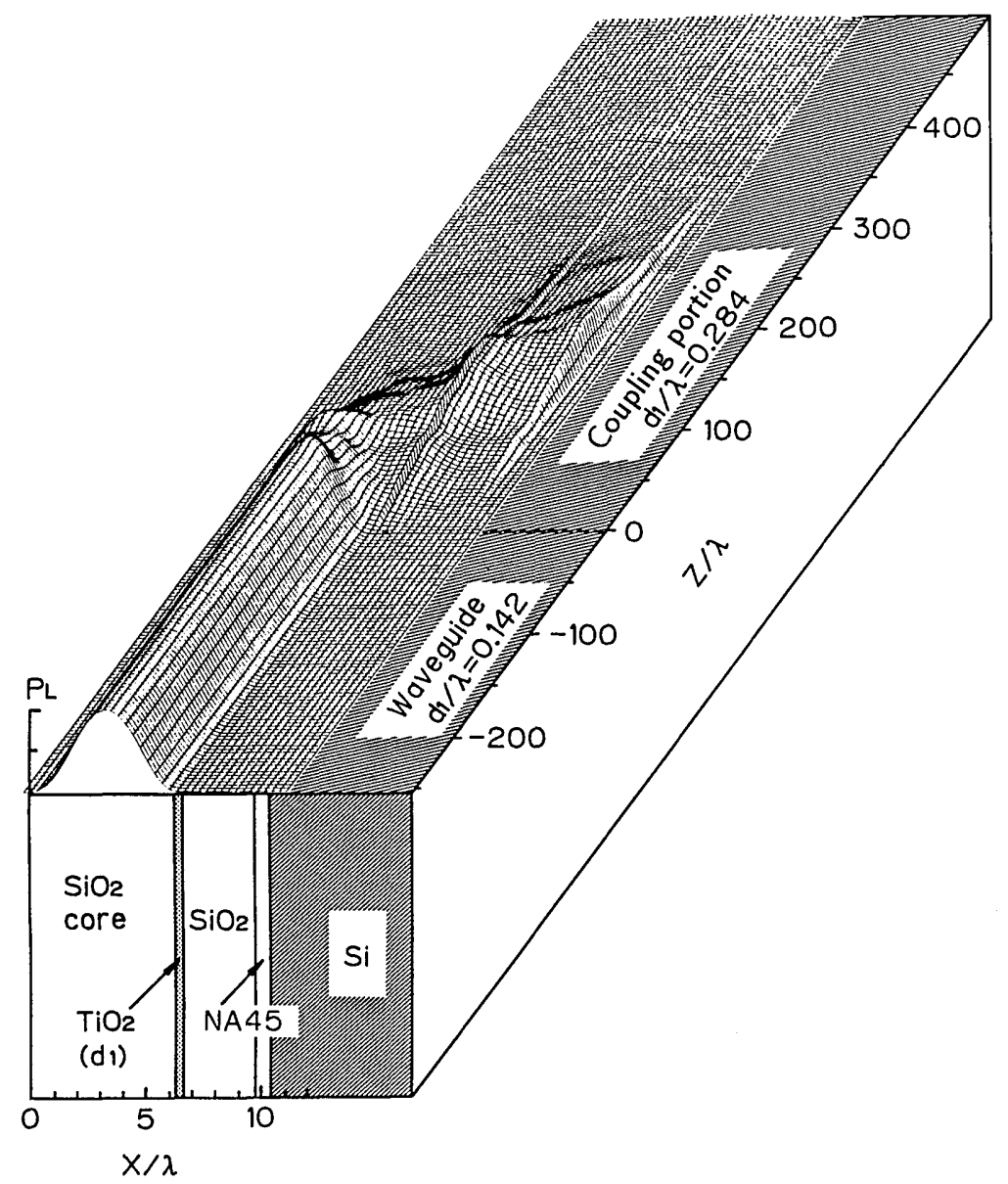

Fig. 9. Simulated light power transition in ARROW integrated structure [c].

[a] with the AR layer. The undesirable length of the almost horizontal part in curve (c) corresponds to the distance over which the radiated light propagates obliquely through the interference cladding. This additional length is usually much shorter than the length of the tapered cladding of a conventional waveguide structure [a]. Thus, curve (a) in Fig. 8(b) should be evaluated by sifting some distance of the tapered cladding to the right, to know the total length of the actual device. In contrast to these structures, the decay in ARROW structure [b] is a little slower than in structure [c], which agrees with the results reported in Sec. III. In any case, in these structures, the loss increases remarkably to a high value from the interference of eigenmodes as well as the effect of the AR layer. Even in structure $[\mathrm{b}]$, a length of no more than $500 \lambda$ is needed to obtain a coupling efficiency $\eta>90 \%$. Taking into account the difficulty of fabrication and the required length of tapered cladding in the conventional structure [a], it should be strongly noted that the two ARROW structures are useful for broad application in integrated optics.

Figure 9 illustrates the simulated transition of light power along the propagation axis in the ARROW structure [c]. The rates of power decay are not constant, because the radiation is expresssed by the superimposition of many eigenmodes and an interference among these modes sometimes enhances the power transition. This is the reason the curves in Fig. 8 are not linear. In the coupling portion with the AR layer having the optimum index and thickness, most reflections at interfaces under the core vanish. Under this assumption, the normalized length of coupling portion $L / \lambda$ required to obtain high coupling efficiency to the photodetector $(>90 \%)$ is given approximately by ${ }^{7}$

$$
\frac{L}{\lambda} \simeq 5 n_{c}\left(\frac{d_{\mathrm{ce}}}{\lambda}\right)^{2}
$$

where the additional length over which light propagates obliquely through the interference cladding is included. When $d_{c} / \lambda=6.3, L / \lambda$ is calculated as 290 . This coincides with the decay length of light power shown in Fig. 9.

\section{Fabrication and Measurement}

To appreciate the loss in the coupling portion of ARROW structure [c], we fabricated a slab ARROW with the first cladding layer having an optimum thickness expressed by Eq. (6). The thickness of each layer was designed for the wavelength of $0.633 \mu \mathrm{m}$ which was used in the measurement. An $\mathrm{SiO}_{2}$ second cladding layer $\left(d_{2}=2 \mu \mathrm{m}\right)$ and an $\mathrm{SiO}_{2}$ core $\left(d_{c}=4 \mu \mathrm{m}\right)$ were made by $\mathrm{RF}$ sputtering. The $\mathrm{TiO}_{2}$ first cladding was made by electron beam (EB) evaporation. Two different thicknesses of the first cladding layer were formed 


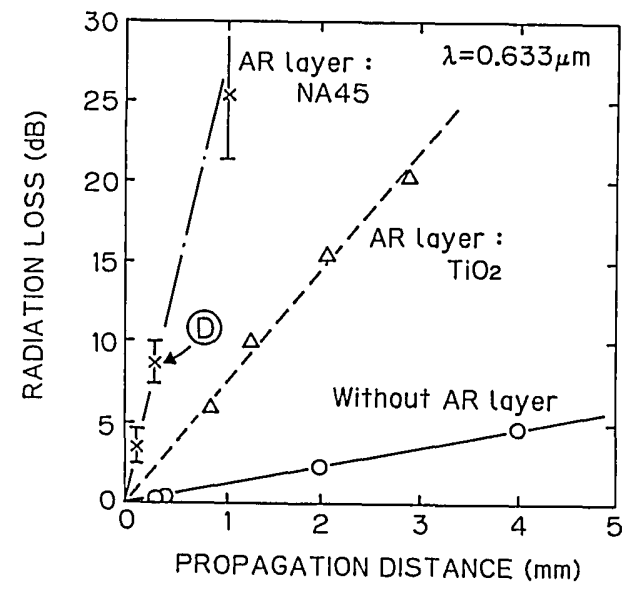

Fig. 10. Measured radiation loss.

by a lift off technique: one was for the waveguide region $\left(d_{1}=0.09 \mu \mathrm{m}\right)$ and the other for the coupling portion $\left(d_{1}=0.18 \mu \mathrm{m}\right)$. Light propagates through the waveguide region and reaches the coupling portion. Various lengths of coupling portion were prepared and the loss was evaluated by the power decay of light passing through this region. Two materials, $\mathrm{TiO}_{2}$ and NA45 glass, were used as the AR layer and results were compared with the case without AR layer.

The measured results are plotted in Fig. 10 where the coupling loss at the input end and the guided loss in the waveguide region are eliminated. Without the AR layer, it requires $\sim 3 \mathrm{~mm}$ to obtain $3 \mathrm{~dB}$ loss $(\eta=50 \%)$. This result agrees with the loss of fundamental mode of ARROW shown in Fig. 3(b). On the other hand, the loss increased rapidly in the case with the AR layer. Even with a $\mathrm{TiO}_{2} \mathrm{AR}$ layer, for which the refractive index is far from the optimum value given by Eq. (13), the radiation loss of coupling portion was seven to ten times as large as that without AR layer. In the case of
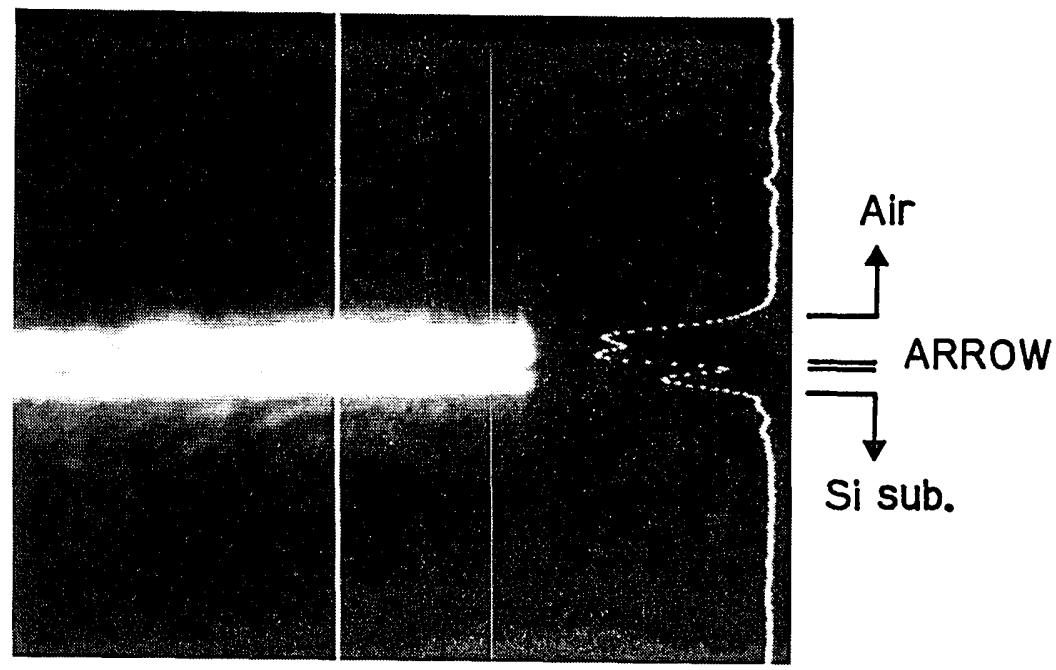

Si sub.

(b)
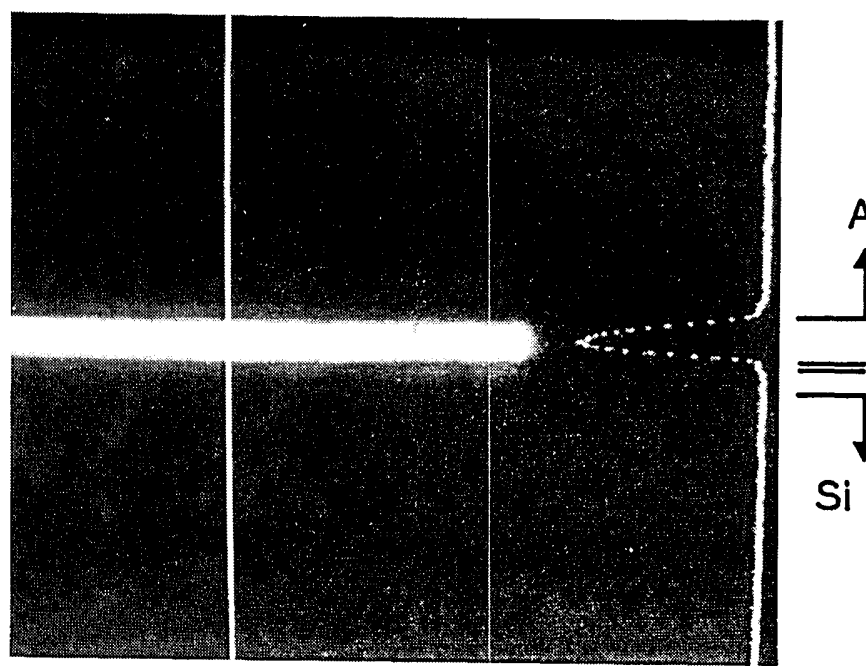

ARROW

Si sub.

Fig. 11. Near field pattern of ARROW: (a) waveguide region $\left(d_{1}=0.09 \mu \mathrm{m}\right)$; and (b) coupling portion $\left(d_{1}=0.18 \mu \mathrm{m}\right)$. 

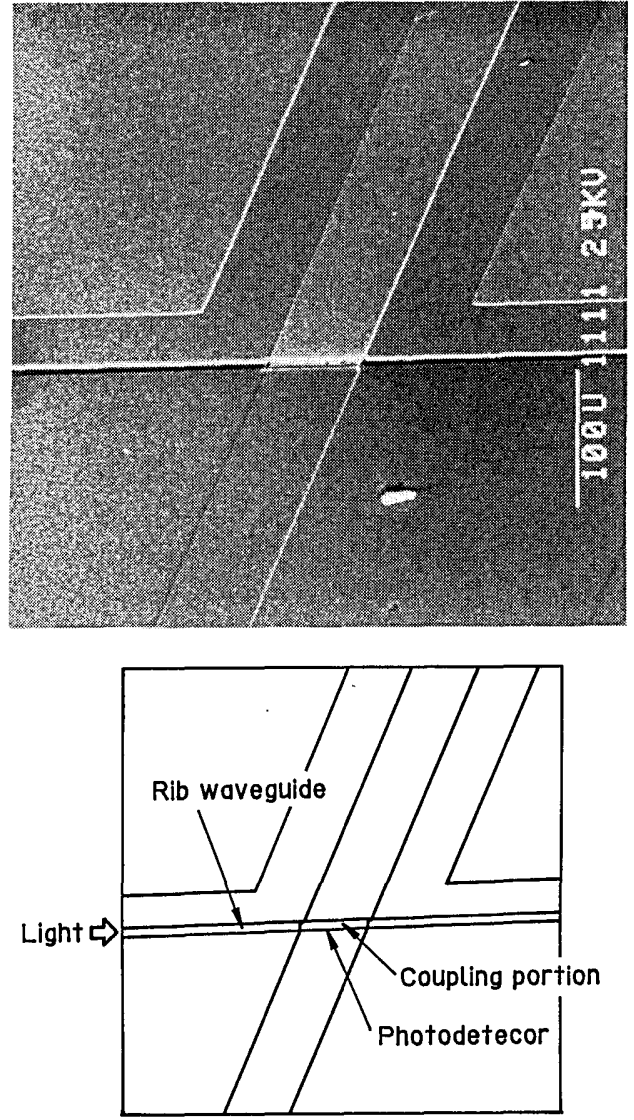

Fig. 12. SEM view of coupling portion of fabricated ARROW integrated device. Light is confined in the lateral direction by ribwaveguide.

the NA45 glass AR layer having nearly optimum index of $1.54,8.2 \mathrm{~dB}$ loss $(\eta=84.9 \%)$ was achieved in the length of $300 \mu \mathrm{m}$. This loss value is a little smaller than the theoretical value in Fig. 8(b). This seems to be caused by the discrepancy between the designed and fabricated thicknesses of the first cladding layer.

Figures 11(a) and (b) show the near field patterns in the low loss ARROW and in the coupling portion indicated by symbol (D) in Fig. 10, respectively. Figure 11(a) displays the ordinary single mode power profile of ARROW. On the other hand, a large amount of light power was observed to leak into the substrate in Fig. 11(b). We integrated a photodetector to ARROW in structure [c]. The external view and the observed output signal for chopped light are shown in Figs. 12 and 13. The demonstration of this type of integrated device is described in Ref. 7.

\section{Vl. Conclusion}

We have analyzed precisely the characteristics of three integrated structures of waveguide and photodetector, i.e., two ARROW types and the conventional waveguide type.

First, we clarified the propagation characteristics of ARROW in detail. We defined the ARROW modes in such a way as to be suitable for practical use and

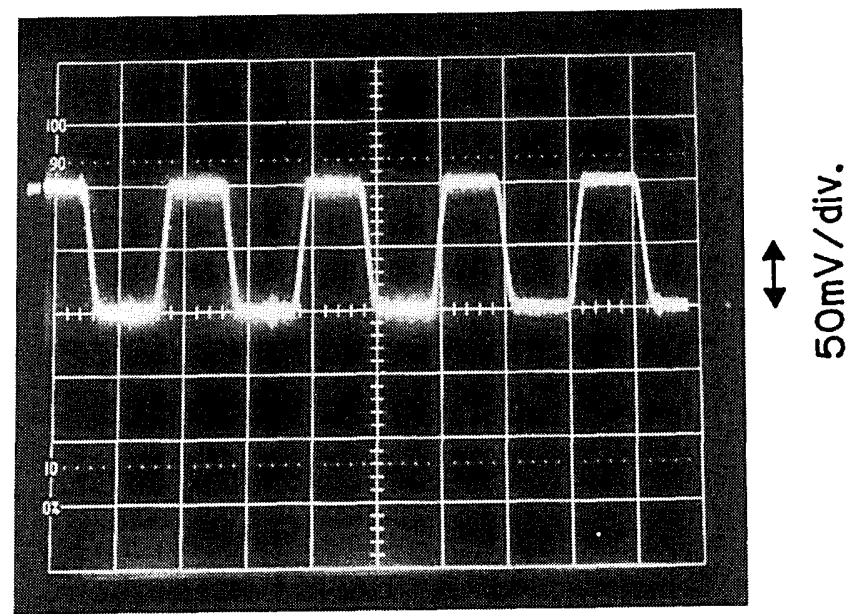

Fig. 13. Output signal of chopped light from fabricated ARROW integrated device.

explained the physical mechanism by which they produce various states of radiation loss as a function of the thickness of the first cladding layer. We derived approximate formulas of the thicknesses of the first cladding layer and of the propagation loss coefficients for two conditions corresponding to low loss propagation and high radiation loss, which can be used for the design of ARROW type integration.

In these integrated structures, the radiation loss which is equivalent to the coupling efficiency from waveguide to photodetector can be improved remarkably by introducing an AR layer formed on the photodetector. By the wave analysis, we showed that the loss becomes $60-70 \times$ as large as that without the AR layer. In particular, this layer is effective for thick core waveguides. Even when $d_{c}=8 \mu \mathrm{m}$ at $\lambda=1.3 \mu \mathrm{m}$ in the conventional waveguide structure, for example, it requires only $120 \mu \mathrm{m}$ to obtain coupling efficiency $>90 \%$. In the ARROW structures, the same amount of coupling efficiency can be achieved. In the experiment, we showed the radiation loss of ARROW to be as high as $8.2 \mathrm{~dB}$ in the coupling portion of $L=300 \mu \mathrm{m}$ with a NA45 glass AR layer when the core thickness is 4 $\mu \mathrm{m}$ at $\lambda=0.633 \mu \mathrm{m}$. This structure requires the additional length of only $\sim 50 \%$ of the detector length, in which light power transits from waveguide core to photodetector. This length is much shorter than that of the tapered cladding in the conventional waveguide structure, which is typically of the order of $1 \mathrm{~mm}$. Thus, the ARROW integrated structure is more suitable for the integration of waveguide and photodetector than is the conventional waveguide.

\section{Appendix A: Derivation of Equation (8)}

Near the cutoff condition of the first cladding modes, the field function in each layer can be expressed by

$$
f(x ; \beta)= \begin{cases}A \sin \left\{\kappa_{c}\left(x+d_{\mathrm{ce}}\right)\right\} & \text { in core } \\ \cos \left(\kappa_{1} x-\phi\right) & \text { in first cladding } \\ B \sin \left\{\kappa_{c}\left(x-d_{1}-d_{\mathrm{ce}} / 2\right)\right\} & \text { in second cladding }\end{cases}
$$


Phase constants in Eq. (A1) are given in terms of the propagation constant $\beta$ by

$$
\begin{aligned}
& \kappa_{c}^{2}=k_{0}^{2} n_{c}^{2}-\beta^{2}, \\
& \kappa_{1}^{2}=k_{0}^{2} n_{1}^{2}-\beta^{2},
\end{aligned}
$$

From the boundary conditions at both interfaces of the first cladding layer, the following relations are obtained:

$$
\begin{aligned}
& \tan \phi=\frac{\kappa_{c}}{\kappa_{1} \tan \left(\kappa_{c} d_{c e}\right)}, \\
& \tan \left(\kappa_{1} d_{1}-\phi\right)=\frac{\kappa_{c}}{\kappa_{1} \tan \left(\kappa_{c} d_{c e} / 2\right)} .
\end{aligned}
$$

Near the cutoff condition for the first cladding modes, the changes of phase in the core and in the second cladding layer are very small, i.e.,

$$
\kappa_{c}=0 \text {. }
$$

By Eq. (A6) and by the assumption that $\kappa_{1} d_{c}$ is much larger than unity, Eq. (A4) is simplified to

$$
\phi \simeq \frac{1}{\kappa_{1} d_{\mathrm{ce}}} .
$$

Similarly from Eq. (A5),

$$
\kappa_{1} d_{1}-\phi-M \pi \simeq \frac{2}{\kappa_{1} d_{\mathrm{ce}}}(M=0,1,2, \ldots) .
$$

From Eqs. (A2), (A3) and (A6), $\kappa_{1}$ is given by

$$
\kappa_{1} \simeq \frac{2 \pi n_{1}}{\lambda}\left[1-\left(\frac{n_{c}}{n_{1}}\right)^{2}\right]^{-1 / 2} \text {. }
$$

By substituting Eqs. (A7) and (A9) into Eq. (A8), we obtain

$$
\frac{d_{1}}{\lambda} \simeq \frac{1}{2 n_{1}}\left[1-\left(\frac{n_{c}}{n_{1}}\right)^{2}\right]^{-1 / 2}\left[M+\frac{3 \lambda}{2 \pi^{2} d_{c} \sqrt{n_{1}^{2}-n_{c}^{2}}}\right] .
$$

\section{Appendix B: Derivation of Equation (10)}

In leaky waveguides, the radiation loss is given by Eq. (3). Now, we consider the light guided through the first cladding layer. On the condition of Eq. (A6), the interference matrix ${ }^{11,14}$ under the first cladding layer is given in terms of parameters in the second cladding layer by

$$
\left[\begin{array}{ll}
m_{11} & m_{12} \\
m_{21} & m_{22}
\end{array}\right]=\left[\begin{array}{cc}
1 & -j k_{0} d_{c e} / 2 \\
0 & 1
\end{array}\right] .
$$

From Eq. (B1), the transmittance through the second cladding layer, $1-R$, is given approximately by ${ }^{11}$

$$
1-R \simeq\left(\frac{2 \lambda}{\pi d_{c e}}\right)^{2} /\left(\sqrt{n_{1}^{2}-n_{c}^{2}} \sqrt{n_{s}^{2}-n_{c}^{2}}\right)
$$

where we have assumed that $k_{0} d_{\mathrm{ce}} / 2$ is much larger than unity. Angle $\theta$ in Eq. (3) is the propagation angle of light through the first cladding layer. This is equivalent to a critical angle of total internal reflection at the boundary between the core and the first cladding layer. This $0_{1}$ is given by

$$
\tan \theta_{1}=\left[\left(\frac{n_{1}}{n_{c}}\right)^{2}-1\right]^{-1 / 2} .
$$

In this condition, the light power spreads from the core to the second cladding layer. Even considering the light through the first cladding layer, the equivalent thickness of core is calculated by integrating power intensity and we obtain Eq. (7). Substituting Eqs. (B2), (B3) into Eq. (3), the maximum loss coefficient is given by

$$
\alpha_{0 \max } \lambda \simeq 0.879\left(\frac{\lambda}{d_{\mathrm{ce}}}\right)^{3} /\left(n_{c} \sqrt{n_{s}^{2}-n_{c}^{2}}\right)(\mathrm{dB} \cdot \lambda / \mathrm{m}) .
$$

\section{Appendix C: Calculation of Excitation Factor $L(\beta)$ and Normalization Factor $N(\beta)$}

Let us suppose both ARROW modes and first cladding modes. We assume the functions of electrical field distribution in the three ranges of propagation constants as follows:

$$
\begin{aligned}
& n_{0}<n_{\text {eq }}<n_{c} \\
& f(x ; \beta)= \begin{cases}C_{0} \exp \left(\gamma_{0} x\right) & \text { in upper cladding } \\
\cos \left(\kappa_{c} x-\phi\right) & \text { in core } \\
C_{1} \sin \left(\kappa_{1} x+\phi_{1}\right) & \text { in first cladding } \\
C_{2} \cos \left(\kappa_{2} x+\phi_{2}\right) & \text { in second cladding } \\
C_{\mathrm{AR}} \sin \left(\kappa_{\mathrm{AR}} x+\phi_{\mathrm{AR}}\right) & \text { in AR layer } \\
C_{s} \cos \left(\kappa_{s} x+\phi_{s}\right) & \text { in substrate }\end{cases} \\
& n_{c}<n_{\text {eq }}<n_{\mathrm{AR}} \\
& f(x ; \beta)= \begin{cases}C_{0} \exp \left(\gamma_{0} x\right) & \text { in upper cladding } \\
C_{c 1} \exp \left(\gamma_{c} x\right)+C_{c 2} \exp \left(\gamma_{c} x\right) & \text { in core } \\
\cos \left(\kappa_{1} x-\phi_{1}\right) & \text { in first cladding } \\
C_{21} \exp \left(\gamma_{2} x\right)+C_{22} \exp \left(-\gamma_{2} x\right) & \text { in second cladding } \\
C_{\mathrm{AR}} \cos \left(\kappa_{\mathrm{AR}} x+\phi_{\mathrm{AR}}\right) & \text { in AR layer } \\
C_{s} \sin \left(\kappa_{s} x+\phi_{s}\right) & \text { in substrate }\end{cases} \\
& n_{\mathrm{AR}}<n_{\text {eq }}<n_{1} \\
& f(x ; \beta)= \begin{cases}C_{0} \exp \left(\gamma_{0} x\right) & \text { in upper cladding } \\
C_{c 1} \exp \left(\gamma_{c} x\right)+C_{c 2} \exp \left(\gamma_{c} x\right) & \text { in core } \\
\cos \left(\kappa_{1} x-\phi_{1}\right) & \text { in first cladding } \\
C_{21} \exp \left(\gamma_{2} x\right)+C_{22} \exp \left(-\gamma_{2} x\right) & \text { in second cladding } \\
C_{\mathrm{AR} 1} \exp \left(\gamma_{\mathrm{AR}} x\right)+C_{\mathrm{AR} 2} \exp \left(-\gamma_{\mathrm{AR}} x\right) & \text { in AR layer } \\
C_{s} \sin \left(\kappa_{s} x+\phi_{s}\right) & \text { in substrate }\end{cases}
\end{aligned}
$$

where the coefficients $C_{1}, C_{2}, \ldots$ and phase shifts $\phi_{1}$, $\phi_{2}, \ldots$ are determined by the boundary conditions. In low loss ARROW, a little light power is radiated from the core so that the field distribution is expressed in terms of several quasiguided modes, ${ }^{13}$ of which propagation constants are expressed by a continuous spectrum having sharp maximums.

With the aid of the characteristic mode expansion, one of the field components $E_{y}(x, z)$ is given by Eq. (15). The excitation factor $L(\beta)$ is determined by the field distribution of incident light $E_{y}(x, 0)$ as

$$
L(\beta)=\frac{\beta}{2 \omega \mu_{0} P} \int_{-\infty}^{\infty} f(x ; \beta) E_{y}(x, 0) d x_{1}
$$




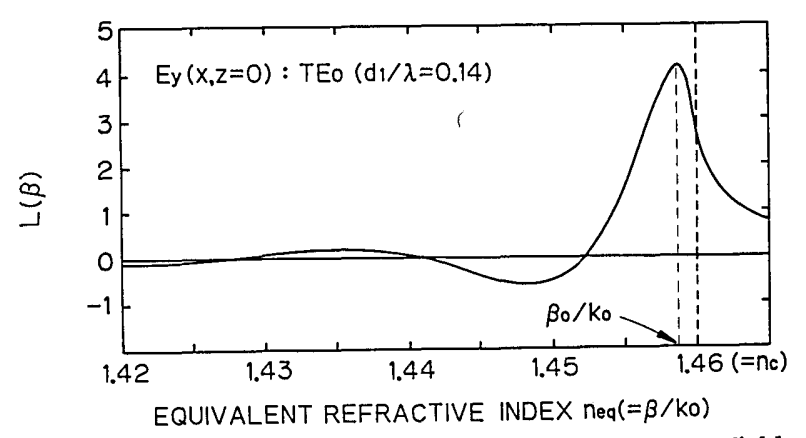

Fig. 14. Calculated excitation factor $L(\beta)$ against input field of profile of low loss ARROW.

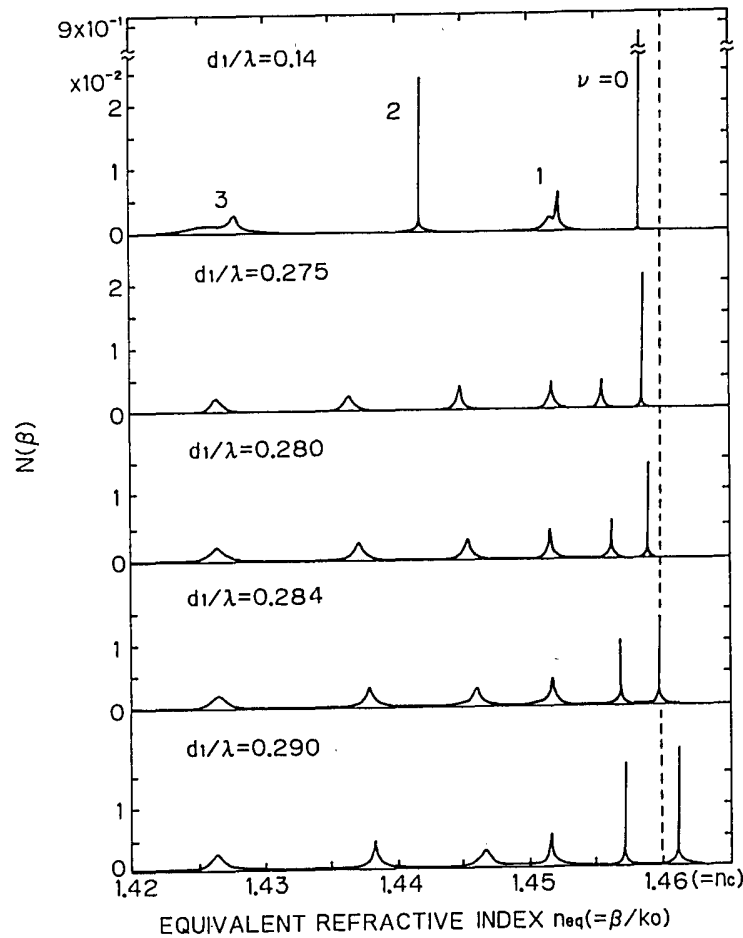

Fig. 15. Calculated normalized factor $N(\beta)$ against various thicknesses of first cladding layer. Mode numbers agree with Fig. $3 . d_{1} / \lambda$ $=0.284$ corresponds to the point (B) in Fig. 3 .

In our case of coupling from ARROW to photodetector, the input field distribution is the guided wave in ARROW. The calculated $L(\beta)$ in the range $1.42<n_{\text {eq }}$ $<1.465$ is shown Fig. 14, where the light power $P$ carried by the mode is assumed to be $1 / 2 \omega \mu_{0}$.

Normalization factor $N(\beta)$ is generally given by

$$
\frac{N^{2}(\beta)}{2 \omega \mu_{0}} \int_{-\infty}^{\infty} f(x ; \beta) f\left(x ; \beta^{\prime}\right) d x=P \delta\left(\beta-\beta^{\prime}\right) .
$$

Substitution of the electric field expressed by Eqs. (31)-(33) into Eq. (C5) gives the function $N(\beta)$ of ARROW as ${ }^{13,14}$

$$
N^{2}(\beta)=\frac{2}{\pi \kappa_{s} C_{s}^{2}}
$$

where we put $P=1 / 2 \omega \mu_{0}$. Similar to $L(\beta)$, the calculated $N(\beta)$ for the various values of $d_{1}$ is shown in Fig. 15. Each sharp maximum indicates the propagation mode, which corresponds to each line in Fig. 3 . When $d_{1} / \lambda$ is 0.284 , which satisfies the condition in the coupling portion, many radiation modes can be seen. Comparing with low loss ARROW $\left(d_{1} / \lambda=0.14\right)$, every peak has a lower height and wide HWHM (half width of half maximum), which means large radiation loss. ${ }^{13,14}$

The transition of light power from the core to the substrate can be calculated by substituting Eqs. (C1)(C6) into Eq. (15). In the case of the ARROW structure shown in Fig. 5(b), the same calculation can be done by substituting zero into $d_{1}$. Also, for the conventional type shown in Fig. 5(a), $d_{1}$ and $d_{2}=0$.

The authors would like to thank K. Iga, Tokyo Institute of Technology, M. Toki and Y. Tsuzuki, Yokohama National University, for support. We thank N. Haneji, H. Watanabe, and M. Hayashi, Yokohama National University, T. Sakaguchi, Tokyo Institute of Technology, H. Nakajima and M. Seino, Fujitsu Laboratories, for help with the experiment.

This work was partly supported by Grants-in-Aid (63460139 and 63790296) from the Ministry of Education, Science and Culture, CASIO Science Promotion Foundation, and Nippon Sheet Glass Foundation for Material Science.

\section{References}

1. J. T. Boyd, C. M. Chuang, and C. L. Chen, "Fabrication of Optical Waveguide Taper Couplers Utilizing $\mathrm{SiO}_{2}$," Appl. Opt. 18, 506-509 (1979).

2. S. Ura, T. Suhara, H. Nishihara, and J. Koyama, "An Integrated-Optic Disk Pickup Device," IEEE/OSA J. Lightwave Technol. LT-4, 913-917 (1986).

3. T. Suhara and H. Nishihara, "Integrated Optics Components and Devices Using Periodic Structures," IEEE J. Quantum Electron. QE-22, 845 (1986).

4. P. Cinguino, C. Cacciatore, C. De Bernardi, F. Genova, M. Puleo, C. Rigo, and A. Stano, "InGaAs PIN Photodiode Monolithically Integrated on InGaAlAs Ridge Waveguides," in Proceedings, Thirteenth European Conference on Optical Communication (Finland, 1987), p. 247.

5. T.L. Koch, P. J. Corvini, W. T. Tsang, U. Koren, and B. I. Miller, "Wavelength Selective Interlayer Directionally Grating-Coupled InP/InGaAsP Waveguide Photodetection," Appl. Phys. Lett. 51, 1060 (1987).

6. M. Erman, P. Jarry, R. Gamonal, J. L. Gentner, P. Stephan, and C. Guedon, "Monolithic Integration of a GaInAs p-i-n Photodiode and an Optical Waveguide: Modeling and Realization Using Chloride Vapor Phase Epitaxy," IEEE/OSA J. Lightwave Technol. LT-6, 399-411 (1988).

7. Y. Kokubun, T. Baba, H. Watanabe and K. Iga, "Monolithic Integration of ARROW-type Demultiplexer and Photodetector," in Proceedings, Fourteenth European Conference on Optics Communication (Brighton, 1988), p. 231; 'T. Baba, Y. Kokubun, and H. Watanabe, "Monolithic Integration of ARROWtype Demultiplexer and Photodetector in Shorter Wavelength Region," IEEE/OSA J. Lightwave Technol. LT-8, 99-104 (1990). 
8. M. McWright Howerton and T. E. Batchman, "A Thin-Film Waveguide Photodetector Using Hydrogenated Amorphous Silicon," IEEE/OSA J. Lightwave Technol. LT-6, 1854-1860 (1988).

9. M. A. Duguay, Y. Kokubun, T. L. Koch, and L. Pfeiffer, "Antiresonant Reflecting Optical Waveguides in $\mathrm{SiO}_{2}-\mathrm{Si}$ Multilayer Structure," Appl. Phys. Lett. 49, 13 (1986).

10. Y. Kokubun, T. Baba, T. Sakaki, and K. Iga, "Low Loss Antiresonant Reflecting Optical Waveguide on a Si Substrate in Visible Wavelength Region," Electron. Lett. 22, 892 (1986).

11. T. Baba, Y. Kokubun, T. Sakaki, and K. Iga, "Loss Reduction of an ARROW Waveguide in Shorter Wavelength and Its Stack
Configuration," IEEE/OSA J. Lightwave Technol. LT-6, 14401445 (1988).

12. Z. Knittl, "Optics of Thin Films," (Wiley, New York, 1976).

13. Y. Suematsu and K. Furuya, "Quasi-Guided Modes and Radiation Losses in Optical Dielectric Waveguides with External Higher Index Surroundings," IEEE Trans. Microwave Theory Technol. MTT-23, 170 (1975).

14. A. K. Ghatak, K. Thyagarajan, and M. R. Shenoy, "Numerical Analysis of Planar Optical Waveguides Using Matrix Approach," IEEE/OSA J. Lightwave Technol. LT-5, 660-667 (1987). 